\title{
Custo-efetividade: comparação entre o modelo \\ "tradicional" e o Programa de Saúde da Família.
}

Cost-effectiveness analysis: comparison of traditional primary health care delivery and the Family Health Program.

Janice Dornelles de Castro* Vanessa da Rocha** Marcia Marinho*** Silvia Pinto****

\section{Resumo}

Este é um estudo de custo-efetividade da provisão da Atenção Básica por meio de duas alternativas de modelos de Atenção Básica - o modelo "tradicional” e o Programa de Saúde da Família. Em um primeiro momento, é feita a discussão teórica sobre avaliação econômica, explicitando toda a sua complexidade e, em um segundo, é realizado um estudo de caso no município de Porto Alegre. Os dados demonstram que o Programa de Saúde da Família foi mais custo-efetivo.

\begin{abstract}
This study analyses the cost-effectiveness of primary health care on the basis of two alternative models of health care delivery - the "traditional" model and the Family Health Program of the Unified Health System. Firstly we make a theoretical approach to health economics assessment in all its complexity, and then we carry out a cost-effectiveness analysis based on a case study in the city of Porto Alegre. The data showed the Family Health Program to be more cost effective.
\end{abstract}

Palavras-chave: Custos de Cuidados de Saúde; Economia da Saúde; Cuidados Primários de Saúde.
Key Words: Health Care Cost; Health Economics;

Primay Health Care.

*Economista, Mestre Health Planning and Financing - London Scholl of Economics, Doutora em Saúde Coletiva - UNICAMPI; Professora da Faculdade de Ciências Econômicas, Universidade Federal do Rio Grande do Sul.

**Administradora hospitalar, UNISINOS, Brasil.

***Mestre em Administração (UFRGS), analista de Sistemas, Datasus, Ministério da Saúde, Brasília, Distrito Federal, Brasil. 


\section{Introdução}

A implantação do Sistema Único de Saúde (SUS) criou a necessidade de reorganização dos serviços de saúde. Neste contexto, o principal desafio, principalmente para os municípios, tem sido a garantia dos princípios básicos da universalidade, da integralidade e da eqüidade. $\mathrm{O}$ aumento de custos do sistema - conseqüência tanto do aumento e envelhecimento da população coberta, como de inovações tecnológicas - criou a necessidade da realização de avaliações dos serviços, com o objetivo de melhor utilizar os recursos escassos. Ou seja, é necessário priorizar o uso dos recursos, buscando não reduzir a oferta e a qualidade dos serviços, e é nesta medida que os estudos de avaliação econômica são importantes, pois colocam à disposição do planejamento os instrumentos necessários para o estabelecimento dessas prioridades.

Este trabalho se propõe a fazer uma avaliação de dois modelos de Atenção Básica - aquele que chamamos de "tradicional" e o Programa de Saúde da Familia -, com o intuito de verificar os custos e a efetividade em cada um deles.

\section{A avaliação econômica}

Para Drumond \& Stoddart ${ }^{1}$, a visão dos economistas sobre a avaliação de custos dos programas de saúde não deve ser apenas aquela dos gastos realizados, mas a do potencial benefício que irão produzir para a sociedade. No entanto, para realizar as avaliações econômicas é inevitável que se trabalhe com a noção de custos de oportunidade, ou do sacrifício, ou escolha entre diferentes alternativas. A noção de custo de oportunidade decorre de uma questão fundamental para a economia: o problema econômico fundamental, ou seja, as necessidades humanas são ilimitadas e os recursos disponíveis são escassos, nesta medida é necessário fazer escolhas sobre o que, para quem, como e quanto produzir. Para tentar responder a essas questões os economistas utilizam a avaliação econômica.

A discussão teórica encontrada na literatura sobre este tema é bastante ambígua, especialmente quando debatem o significado dos diferentes tipos de avaliação econômica: custo-benefício (ACB), custo-utilidade (ACU), cus- to-efetividade (ACE) e custo-mínimo (ACM). Drumond \& Stoddart, em seu artigo Principles of economic evaluation of healh programmes ${ }^{1}$, colocam pontos importantes que devem ser considerados, ao proporem estes tipos de estudos, sugestão corroborada por inúmeros autores ${ }^{2,3,4,5,6,7}$.

O primeiro ponto levantado diz que uma avaliação econômica sempre é uma comparação entre mais de uma alternativa de possibilidades de ação - não se esquecendo de que uma das alternativas pode ser "o não fazer nada". Os estudos em que não são realizadas as comparações, não são de avaliação econômica e podem ser chamados de descrição de custos ou de descrição de resultados.

O segundo ponto é o estabelecimento, tecnicamente, da efetividade dos programas ou ações que estão sendo avaliados, uma vez que a falta das evidências clínicas de efetividade afetam a avaliação econômica. No entanto, sabemos que, quando os programas ou as ações possuem objetivos amplos, caso da maioria das intervenções de saúde pública, o estudo fica mais complexo. Para Mills ${ }^{4}$, o estabelecimento da efetividade pode ser feito por meio de ensaios clínicos controlados, no entanto, afirma que este tipo de estudo é muito caro, e muitas vezes os resultados são inconclusivos, pois é muito difícil estabelecer a relação entre as atividades de saúde e suas conseqüências. Portanto, sugere que sejam utilizadas como medidas de efetividade: (a) mudanças nas taxas de prevalência ou incidência, numa determinada população, em um dado período de tempo, analisando antes e após a intervenção avaliada; (b) definição da efetividade de uma intervenção obtida por consenso dos profissionais após a utilização método Delphi; (c) revisão das evidências de efetividade por meio de publicações.

Outro ponto levantado é a necessidade de identificação de todos os custos e conseqüências importantes para o estudo. Devem ser considerados os custos diretos - representados pelo custo de organizar e operar o sistema de saúde e pelas despesas que os pacientes e suas famílias tiverem com o tratamento -, assim como os custos indiretos, que são relativos ao tempo de trabalho perdido e os custos físicos, resultantes do sofrimento causado pelo trata- 
mento. Por fim, devem-se considerar os custos produzidos fora do sistema de saúde, como restrições criadas pelos governos à produção de medicamentos.

Em relação às conseqüências, devem ser consideradas as mudanças físicas, emocionais ou de funcionamento que resultem em poupança de recursos para o sistema e para os pacientes e suas famílias, assim como a diminuição no tempo de trabalho perdido, e, por fim, as mudanças positivas na qualidade de vida dos pacientes e suas famílias $^{1}$. Para Mills ${ }^{3}$, poucos estudos em países do terceiro mundo levam em consideração as conseqüências emocionais e sociais da doença ou as mudanças na qualidade de vida dos pacientes e suas famílias, no entanto, existem muitos estudos sobre as conseqüências em relação às mudanças físicas.

Além dos pontos discutidos acima, os autores ${ }^{1}$ sugerem que devem ser utilizadas unidades de medidas de custos e conseqüências de forma apropriada - por exemplo, se uma ação é parte de uma atividade maior, como atribuir a proporção correta dos custos e conseqüências a ela?

Segundo Mills ${ }^{3}$, o maior problema no terceiro mundo é a inexistência rotineira de informações, tanto de custos, quanto de efetividade que sejam confiáveis, especialmente as informações sobre os efeitos ou impacto das ações de saúde. Sendo assim, sugere que sejam utilizadas as medidas de processo ou intermediárias, como número de pacientes tratados, número de vacinas realizadas. Mas é preciso não esquecer que assumir medidas de utilização, enquanto medidas de efeitos, pode provocar distorções no estudo.

Os autores Drumond \& Stoddart ${ }^{1}$ também sugerem que se devem utilizar medidas adequadas de valoração de custos e conseqüências. Por exemplo, para avaliar os custos diretos de uma ação de saúde, poderia ser usado os preços de mercado desta ação, que, nas economias desenvolvidas, corresponderá aproximadamente aos custos de produção. Mas isso não ocorre nos países subdesenvolvidos por inúmeras razões, tais como a existência de tarifas, impostos e subsídios, assim como as altas taxas de desemprego e subemprego que afetam os preços dos bens e serviços domésticos, em relação aos preços internacionais ${ }^{3}$. A sugestão é ajustar os preços para os do mercado inter- nacional, criando preços novos, que são chamados de preços-sombra.

Outro ponto levantado refere-se ao fato que cada tipo de avaliação econômica irá considerar as conseqüências de diferentes formas. $\mathrm{Na}$ forma mais simples de estudo, chamada de análise de custos, espera-se, ou se assume, com base em alguma evidência (estudos anteriores que confirmem a efetividade clínica das diferentes ações), que as conseqüências ou os benefícios serão iguais; por isso, são considerados apenas os custos das diferentes alternativas de ação. No entanto, é importante observar que este é um tipo de avaliação econômica incompleta ${ }^{1,2,3,5,6,7}$.

Existem outras três formas de avaliação econômica completa, ou seja, aquelas que comparam custos e resultados, e que têm em comum o fato de que os custos são expressos em unidades monetárias e os benéficos em unidades de conseqüência. Essas análises são comumente classificadas sob o genérico título de análises de custoefetividade $^{1}$, no entanto, existem algumas diferenças importantes entre elas.

O tipo mais simples é chamado de análise de custo-mínimo, neste caso, as conseqüências dos diferentes tipos de ações são avaliadas por estudos clínicos controlados, não são assumidos como dados, e, normalmente, a avaliação econômica é realizada ao mesmo tempo. Se os resultados forem equivalentes para todas as conseqüências, então se realiza a análise de custos. No entanto, é incomum que existam, pelo menos, dois tipos de intervenção que possuam exatamente as mesmas conseqüências ${ }^{1,8}$.

Outro tipo são os estudos de custo-efetividade propriamente ${ }^{1,2,3,5,7,8,9}$, cuja característica é ter, pelo menos, uma dimensão importante das conseqüências dos diferentes tipos de ação em que a comparação pode ser feita. A extensão da vida é uma conseqüência importante, assim as diferentes alternativas de ação podem ser medidas pelo "custo por ano de vida ganho”, por exemplo. Em alguns casos, é aceitável a utilização de medidas de resultado intermediárias, ou de processo, como são mais conhecidas, como o "número de casos diagnosticados corretamente", pois é possível inferir a relação entre esta conseqüência com os 
benefícios ou resultados de impacto, para o paciente 1,3,7,8. $^{1,2}$

Podemos dizer que o custo-efetividade procura avaliar se os objetivos foram alcançados de forma eficiente e eficaz. Um projeto pode ser eficiente quando opera com custos mínimos; por outro lado, ele pode não ser eficaz, pois não atinge aqueles grupos selecionados inicialmente. Sendo assim, é preciso medir a eficácia para conhecer o seu impacto no grupo selecionado, e, neste caso, os custos serão expressos em unidades monetárias e a efetividade, pelo número de "vidas salvas' ou qualquer outro objetivo relevante" alcançado ${ }^{1,3,7,8}$.

Uma característica importante da análise de custoefetividade é a possibilidade de hierarquizar as diferentes opções de ações para se alcançar os objetivos do projeto, analisando os custos de cada uma para obter uma unidade de produto. $\mathrm{Na}$ análise de custo-efetividade, os objetivos do projeto não são avaliados, pois são consideradas questões do campo político, "são os fins procurados pela sociedade e expressos por aqueles que assumem sua representação" e, portanto, estão dados.

Sendo assim, estabelecidos determinados objetivos para um projeto social, a análise de custo-efetividade busca avaliar as diferentes alternativas possíveis para alcançálos, que devem ser comparáveis entre si, e, nesta medida, é fundamental que as alternativas tenham a mesma população-alvo.

Cohen e $\mathrm{Franco}^{7}$ afirmam que as análises de custo-efetividade são vinculadas à eficiência operacional, e, neste caso, são avaliações de processo, e não de impacto, bastando selecionar a alternativa que minimiza os custos, pois os objetivos finais do projeto e a unidade de produto se confundem. Esta discussão traz à tona duas questões importantes: (a) a maioria dos projetos sociais possui múltiplos objetivos e existem fatores externos à eficiência operacional que podem afetar o seu alcance; (b) os objetivos são determinados politicamente, e pressupõe-se que tenham sido bem selecionados.

Sobre este tema, Mills. \& Gilson ${ }^{8}$ afirmam que, normalmente, os programas de Atenção Primária em Saúde, possuem objetivos amplos e com efeitos que não po- derão ser medidos por apenas uma unidade de efeito. Para superar este problema podem ser usadas unidades de resultado intermediário ou de processo, como número de crianças imunizadas ou proporção de mulheres grávidas que tiveram acesso a consultas de pré-natal. Essas medidas são de acesso, cobertura e de realização de metas, e isso poderá avaliar a capacidade do programa de saúde, aumentar a provisão dos serviços. Não informarão se este programa é a melhor escolha, mas a melhor maneira de prestar um serviço que foi anteriormente escolhido como adequado $^{8}$.

O terceiro e último tipo de estudo em que os custos são expressos em unidades monetárias e os benéficos em unidades de conseqüência são as análises de custo-utilidade considera a qualidade ou utilidade "por ano de vida ganho" após a intervenção e leva em consideração a morbidade associada à extensão da vida. As alternativas de ação são comparadas em termos de custo por qualidadeajustada por ano de vida, mais conhecida por QALYS ${ }^{1,3,7,8}$.

Por fim, existe outro tipo de estudo de avaliação econômica em que os custos e conseqüências são expressos em unidades monetárias, são os estudos de custo-benefício, que permitem comparar diferentes alternativas que possuam diferentes objetivos, como, por exemplo, programas da área da saúde com programas da área de transportes.

No entanto, é difícil atribuir um valor monetário às conseqüências de um programa de saúde; por isso, a utilização deste método fica restrita. Serve aos estudos em que pode ser atribuído, facilmente, um valor monetário às conseqüências, como, por exemplo, o aumento da produtividade ou a volta ao trabalho decorrente da cura de um paciente, ou, ainda, o aumento do período de vida produtiva, e, também, os estudos em que se avalia a poupança para o sistema de saúde decorrente da cura ou melhora nas condições de saúde do paciente ${ }^{1,7,8}$.

A avaliação social de projetos ou atividades em seu sentido mais amplo pretende medir o impacto do projeto sobre o nível de bem-estar socioeconômico do país ${ }^{1}$. A eficiência é uma relação entre os produtos e seus custos; 
no entanto, os projetos, tanto econômicos como sociais, são extremamente complexos, pois buscam alcançar mais de um objetivo, por meio da execução de inúmeras atividades que consomem diversos tipos de insumos, e todas essas questões devem ser levadas em consideração no momento da avaliação da eficiência de um projeto. A discussão teórica metodológica sobre a avaliação econômica, especialmente na área da saúde, ainda tem muitos caminhos a percorrer. Esperamos, aqui, contribuir para a melhor compreensão do significado e da importância destes estudos.

\section{Indicadores de efetividade}

Para a avaliação da efetividade é necessário definir os indicadores que vão medir o sucesso dos objetivos. Os indicadores devem ser confiáveis, ou seja, diferentes avaliadores devem poder obter os mesmos resultados e devem medir precisamente aquilo que se deseja - preferencialmente, devem medir mudanças específicas que possam ser atribuídas ao projeto, e não a outras variáveis ${ }^{1,10}$.

Alguns indicadores têm sido muito utilizados na área da saúde e formam algumas grandes categorias, tais como: os indicadores de oferta, de acesso e utilização de serviços de saúde, de qualidade da atenção, da situação da saúde e das condições de saúde, e, ainda, os de financiamento. Para cada uma dessas grandes categorias, podem ser utilizados diferentes indicadores ${ }^{11}$. Neste estudo trabalhamos com três categorias de indicadores: oferta, qualidade e acesso/utilização.

\section{O sistema de cálculo dos custos}

Existem diferentes formas de calcular os custos das atividades ${ }^{10}$ : são os sistemas de custeio total e parcial, por absorção ou tradicional, o variável ou direto ou marginal, por atividade, por taxas, por procedimento, por patologia ou ainda o custo padrão. $\mathrm{O}$ sistema de custeio por absorção é considerado um sistema de custeio integral, pois ele apropria todos os custos incorridos para a produção de um bem ou serviço; ou seja, considera os custos diretos, indiretos, fixos e variáveis, sem considerar, no entanto, os custos para os pacientes. A primeira condição para a utilização deste sistema de custeio é a setorização ou divisão da instituição em centros de custos que devem corresponder ao conjunto de atividades necessárias para que ocorra a produção do bem ou serviço que está sendo avaliado. Os centros de custos expressam a despesa e a sua relação com o bem ou serviço que está sendo avaliado; por isso, são classificados como diretos ou produtivos aqueles que possuem uma relação direta com a produção do bem ou serviço, e como indiretos, administrativos ou de apoio aqueles que realizam despesas para a produção do bem e serviço analisado, mas também para a produção de outros bens e serviços. Os custos indiretos devem ser rateados com base no plano de centros de custos da instituição, assim os custos dos centros de custos de apoio e administrativos serão rateados entre todos os centros de custos, utilizando critérios arbitrários e apropriados ao custo final do produto ou serviço. A principal desvantagem deste sistema é a realização dos rateios usando critérios arbitrários, o que pode implicar na avaliação incorreta dos custos finais ${ }^{12,13}$.

Escolhemos esta metodologia, pois sabemos que os custos indiretos não são a parcela mais importante dos custos para a Atenção Básica da saúde em Porto Alegre, o que foi constatado em estudos anteriores ${ }^{10,14}$. Além disso, existe a facilidade de adaptar o formato de centros de custos à estrutura do orçamento da Secretaria Municipal de Saúde de Porto Alegre, o que simplifica a coleta das informações.

\section{Objetivo geral}

Fazer uma análise de custo-efetividade da Atenção Básica, comparando duas alternativas de provisão de serviços de Atenção Básica - modelo "tradicional” e Programa de Saúde da Família - a partir do estudo de caso de duas unidades de Atenção Básica de saúde em Porto Alegre, no ano de 2002.

\section{Aspectos metodológicos}

Os estudos de custo-efetividade são mais adequados para comparar duas políticas que buscam o mesmo 
objetivo, analisando os custos das diferentes alternativas; neste caso, o objetivo é a provisão de serviços de Atenção Básica e as alternativas são os dois modelos de Atenção Básica: Programa de Saúde da Família (PSF) e tradicional. Qual deles será o mais custo-efetivo? Qual deveria ser escolhido por ser capaz de atingir o objetivo a um menor custo ${ }^{1,7,10,15}$ ?

Este estudo de caso pretende responder a essas questões, avaliando duas unidades básicas, uma do PSF e outra do modelo tradicional, em Porto Alegre, no ano de 2002.

Os serviços públicos de saúde do município estão divididos em duas áreas principais: a vigilância da saúde e a assistência à saúde, que abriga os serviços de atenção ambulatorial básica, em que estão vinculadas as unidades de saúde e as unidades do Programa de Saúde da Família.

A cidade está dividida em distritos de saúde, cada qual com uma gerência responsável pela organização dos serviços na região. As unidades básicas de saúde devem ser a porta de entrada no sistema. Nas unidades, com modelo de atenção que estamos chamando de tradicional, são oferecidos os serviços de uma equipe multidisciplinar composta por médicos (clínico geral, ginecologista e pediatra), enfermeiros, auxiliares de enfermagem e nutricionistas. E, nas unidades de saúde do PSF, a equipe é composta por médico generalista, enfermeiros, auxiliares de enfermagem e agentes comunitários. Além disso, é definida a área de abrangência, e as famílias da região são cadastradas. O tipo de atenção à saúde prestado nas duas formas de organização das unidades básicas são similares ${ }^{10}$.

Para o levantamento dos dados de custos foi utilizada a metodologia de apropriação de custos por absorção ${ }^{10,12,14}$ adaptada para este estudo. Os dados de despesa com Serviços de Terceiros foram obtidos na execução orçamentária, e, então, foi calculado o rateio, com base no número de funcionários, para cada centro de custos. $\mathrm{O}$ gasto com material de consumo foi obtido nos relatórios do Almoxarifado, considerando os itens entregues em cada unidade de saúde, e os dados com despesas de pessoal foram obtidos no setor de recursos humanos, não havendo necessidade de realizar rateios para esses itens.

Foram estudados os diferentes indicadores de efetividade das políticas públicas ${ }^{7,11,15,16}$ e selecionados aqueles que poderiam melhor demonstrar a efetividade dos dois modelos de atenção. Como indicador de oferta usamos o total de atendimentos (procedimentos e consultas médicas) per capita; como indicador da qualidade da atenção foi utilizado o total de atendimentos pré-natal por gestante e, enquanto indicador de acesso/utilização dos serviços, usamos a cobertura vacinal $1^{1,3,7,8}$.

O período definido para este estudo foi do ano de 2002 e a escolha das duas unidades básicas foi feita pelos pesquisadores em conjunto com a equipe da Secretaria Municipal de Saúde, usando como critério a semelhança nas suas características.

\section{Resultados}

A população das duas unidades eram bastante parecida em termos numéricos (7.062 e 7.093 pessoas), assim como as características epidemiológicas e socioeconômicas. Além disso, as unidades selecionadas prestavam o mesmo tipo de assistência à população: atenção básica. No entanto, em relação à composição de suas equipes, elas diferiam um pouco, uma delas, a unidade do PSF, trabalhava com dois médicos, dois enfermeiros, quatro auxiliares de enfermagem, oito agentes comunitários e um estagiário; a outra, a unidade tradicional, possuia na sua equipe: cinco médicos, um enfermeiro, seis técnicos de enfermagem.

Observamos que, para o PSF, o custo total foi de $733.170,43$ reais e, para a unidade tradicional, foi de 852.046,06 reais. O custo per capita foi de 103,82 reais e 120,12 reais, respectivamente (Tabela 1 ).

Podemos observar que o PSF apresentava os custos menores nas categorias analisadas. Os custos diretos eram menores: essa não é uma surpresa, pois a maior parcela dos custos diretos é representada pelos custos de pessoal, e, apesar de no PSF os salários serem maiores, a unidade tradicional possui um número de trabalhadores muito maior. Mas será que esses trabalhadores conseguem ter um trabalho de melhor qualidade e em maior quantidade? Para avaliar a efetividade dos modelos de atenção utilizamos indicadores de oferta, de qualidade e de acesso 
ou utilização. $\mathrm{Na}$ tabela 2 apresentamos os dados para a análise do custo-efetividade dos dois modelos de atenção básica.

Os dados desta tabela nos informam que o indicador de oferta, usando o "total de atendimentos (procedimentos e indicador é maior para a unidade tradicional para todas as vacinas, exceto a tetravalente. Devemos ainda considerar que o custo total per capita do PSF foi de 103,82 reais ao ano e, para a unidade tradicional, foi de 120,12 reais ao ano.

Tabela 1: Custos das unidades básicas de saúde: USF e UBS - Porto Alegre 2002:

\begin{tabular}{lcccc}
\hline & Custos diretos & Custos indiretos & Custo total & Custo per capta \\
\hline $\begin{array}{l}\text { Unidade básica do Programa } \\
\text { de Saúde da Família }\end{array}$ & $661.482,51$ & $71.687,92$ & $733.170,43$ & 103,82 \\
\begin{tabular}{l} 
Unidade básica tradicional \\
\hline
\end{tabular} & $767.707,33$ & $84.338,73$ & $852.046,06$ & 120,12 \\
\hline
\end{tabular}

Fonte: Secretaria Municipal de Saúde de Porto Alegre, 2002.

Tabela 2: Comparação de indicadores de efetividade e do custo per capta, USF e UBS - Porto Alegre, 2002:

\begin{tabular}{|c|c|c|c|}
\hline $\begin{array}{l}\text { Indicador } \\
\text { efetividade }\end{array}$ & Descrição & $\begin{array}{c}\text { Unidade básica } \\
\text { Programa de Saúde } \\
\text { da Família }\end{array}$ & $\begin{array}{l}\text { Unidade básica } \\
\text { Tradicional }\end{array}$ \\
\hline Oferta & $\begin{array}{c}\text { Total de atendimentos } \\
\text { (procedimentos e } \\
\text { consultas médicas) } \\
\text { percapita }\end{array}$ & 3,75 & 2,3 \\
\hline Qualidade & $\begin{array}{l}\text { Total de atendimentos } \\
\text { pré-natal realizados }\end{array}$ & 33,5 & 20,5 \\
\hline \multirow[t]{6}{*}{ Acesso / utilização } & Cobertura vacinal & & \\
\hline & Sabin & $110,39 \%$ & $118,66 \%$ \\
\hline & Tetravalente & $133,38 \%$ & $120,51 \%$ \\
\hline & Sarampo & $85,09 \%$ & $133,49 \%$ \\
\hline & Hepatite B & $101,18 \%$ & $100,12 \%$ \\
\hline & BCG & $133,38 \%$ & $169,34 \%$ \\
\hline Custo per capta & & 103,82 & 120,12 \\
\hline
\end{tabular}

Fonte: Secretaria Municipal de Saúde de Porto Alegre, 2002.

consultas médicas) per capita" é maior para o PSF, ou seja, foram feitos 3,75 atendimentos per capita no ano, enquanto que, para a unidade tradicional, foram realizados 2,3 atendimentos per capita no ano. Com relação ao indicador de qualidade, usando o "atendimento pré-natal por gestante", ocorre o mesmo, o PSF registra um número maior que a unidade tradicional. São 33,5 atendimentos por gestantes contra 20,5. Por fim, analisamos o indicador de acesso/utilização, usando a "cobertura vacinal". Neste caso, o
Sendo assim, podemos afirmar com base neste estudo que o modelo de atenção do PSF foi mais custoefetivo, ou seja, a um custo menor, realizou um número maior de atividades, e com maior qualidade destes atendimentos, tanto quando observamos do ponto de vista do indicador de oferta, quanto do indicador de qualidade. Apenas em relação ao acesso e utilização os dois modelos têm comportamento semelhante. 


\section{Conclusões}

Os resultados parecem indicar que o modelo de atenção do Programa da Saúde da Família está garantindo a prestação de serviços com menor custo e maior efetividade. No entanto, esses são resultados de uma pesquisa piloto, que avaliou apenas duas unidades, durante um ano, e utilizou três indicadores de efetividade.

Sabemos que, dentre os custos da atenção básica em Porto Alegre ${ }^{10,14}$, os custos com Pessoal representam os maiores gastos. Também sabemos que os salários dos profissionais do PSF são maiores que dos profissionais da unidade tradicional - nesse sentido, era de se esperar que os custos do PSF fossem mais elevados. No entanto, esse fator é compensado pelo maior número de profissionais na unidade tradicional, que, mesmo assim, realizaram um número menor de atendimentos per capita e também um número menor de atendimentos pré-natal por gestante, apenas em relação à cobertura vacinal o comportamento foi similar para os dois modelos estudados.

\section{Referências}

1. Drumond MF, Stoddart GL. Principles of economic evaluation of health programmes. Wid hith statist quart. 1985;38:355-367.

2. Green A, Barker C. Priority setting and economic appraisal: whose priorities - the community or the economist? Soc Sci Med. 1988;26(9):919-929.

3. Mills A. Economic evaluation of health programmes: application of the principles in developing countries. Wid hith statist quart. 1985;38:368-381.

4. Mills A. Survey and examples of economic evaluation of health programmes in developing countries. Wid. hith. statist.quart. 1985;38:402-430.

5. Torrance GW. Measurement of health state utilities for economic appraisal. Journal of Health Economics. 1986;5:130 .

6. Van Norren B, Boerma JT, Sempebwa EKN. Simplifying the evaluation of primary health care programmes. Soc Sci Med. 1989;28(10):1091-1097.

7. Cohen E, Franco R. Avaliação de projetos sociais.
Petrópolis (RJ): Vozes; 1994.

8. Mills A, Gilson L. Health economics for developing countries: a survival kit. London: London School of Hygiene and Tropical Medicine; 1988 Summer 1988. Report No.: 17.

9. Silva LK. Avaliação tecnológica e análise custo-efetividade em saúde: a incorporação de tecnologias e a produção de diretrizes clínicas para o SUS. Ciência e Saúde Coletiva. 2003;8(2):501-520.

10. Castro JD. A utilização do sistema de custeio por absorção para avaliar custos da atenção básica de saúde:reformulações e aprimoramentos metodológicos. Campinas (SP): Universidade Estadual de Campinas; 2000. 11. Nunes A, Santos JRS, Barata RB, Vianna S. Medindo Desigualdades em Saúde no Brasil: uma proposta de monitoramento. Brasília (DF): OPAS, OMS, IPEA; 2001.

12. Beulke R, Bertó DJ. Gestão de custos e resultados na saúde: hospitais, clínicas, laboratórios e congêneres. São Paulo: Saraiva; 1997.

13. Conselho Regional de Contabilidade do Estado de São Paulo C. Custo como ferramenta gerencial. São Paulo: Atlas; 1995.

14. Castro JD. Análise da evolução dos custos do Programa de Saúde da Família de Porto Alegre no período de 1998 e 2002. In: ABRASCO, editor. VIII Congresso Brasileiro de Saúde Coletiva; 2003; Brasília: ABRASCO; 2003. 15. Piola S, Vianna S. Economia da Saúde: conceitos e contribuições para a gestão em saúde. Brasilia (DF): IPEA; 1995. 16. Hartz MA, Pouvouville G. Avaliações dos programas de saúde: a eficiência em questão. Ciência e Saúde Coletiva 1998;7:68-82.

\section{Endereço para correspondência:}

Rua Furriel Luis Antonio Vargas 106/404

Porto Alegre - RS

CEP: $90.470-130$

\section{Endereço eletrônico:}

janice@fiocruz.br 